\title{
Eliminação do desbaste na cultura do algodoeiro(1)
}

Edivaldo $\mathrm{Cia}^{(2)}$, Enes Furlani Júnior ${ }^{(3)}$, Nelson Paulieri Sabino(2), Milton Geraldo Fuzatto ${ }^{(4)}$, Carlos Antonio Menezes Ferraz ${ }^{(4)}$, Luiz Henrique Carvalho ${ }^{(4)}$, Nelson Bortoletto ${ }^{(5)}$, José Carlos Sabino ${ }^{(5)}$ e Mario Pércio Campana ${ }^{(5)}$

Resumo - Este trabalho foi desenvolvido nos anos agrícolas de 1979/80 a 1982/83 em diferentes localidades no Estado de São Paulo, com o objetivo de avaliar o efeito da eliminação do desbaste na cultura do algodoeiro Gossypium hirsutum L. O delineamento experimental empregado foi o de blocos ao acaso, em esquema fatorial, com seis tratamentos e seis repetições. Os tratamentos foram constituídos de 12 e 24 sementes $/ \mathrm{m}$, submetidas ao deslintamento mecânico, deslintamento mecânico + tratamento com fungicidas e deslintamento químico + tratamento com fungicidas. Para a avaliação dos resultados foram feitas duas análises conjuntas, com base na emergência das plantas: emergência normal e emergência tardia, estudando-se as características: emergência, produção, estande final, peso de capulho e semente, porcentagem e índice de fibra. Os resultados mostraram que em condições edafoclimáticas adversas à germinação ocorreu baixa emergência, causando, com isso, menor população de plantas, e, conseqüentemente, prejuízos à produção de algodão. Com maior estande ocorreram menores pesos de capulho e de sementes, e menor porcentagem e índice de fibra. Constatou-se que existe a viabilidade de eliminação da prática do desbaste por meio do tratamento das sementes com fungicidas aliado ao deslintamento químico, desde que o plantio seja efetuado em boas condições de ambiente à germinação.

Termos para indexação: algodão, população de plantas, tratamento químico de sementes, deslintamento, raleio, métodos mecânicos.

\section{Elimination of thinning practices on cotton crop}

Abstract - This work was developed during the agricultural years of 1979/80 to 1982/83 in different localities in the State of São Paulo, Brazil, to evaluate the effect of plant thinning in the cotton plantation. The trial was carried out in a randomized block design and in a factorial scheme with six treatments and six replicates. The treatments consisted of 12 and 24 seeds $/ \mathrm{m}$ submitted to mechanical delinting + fungicide, and chemical delinting + fungicides. For evaluation of the results, two conjunct analyses were done based on the plant emergence: normal emergence and late emergence, by studying the following traits: emergence, production, final stand, boll weight, seed weight and fiber index. The results showed that under adverse conditions to germination a low emergence occurred, causing smaller plant population and consequently lesser cotton production. With greater stand, smaller boll weight, seed weight, and minor percentage and index of fiber occurred. It was evidenced that the viability of eliminating the plant population thinning by seed treatment with fungicides and chemical delinting exists, since their planting be done under good environment conditions for germination.

Index terms: plant population, seed dressing, delinting, mechanical methods.

(1) Aceito para publicação em 27 de outubro de 2000 .

(2) Instituto Agronômico (IAC), Centro de Algodão e Fibrosas Diversas, Caixa Postal 28, CEP 13020-902 Campinas, SP. Bolsista do CNPq. E-mail: cia@cec.iac.br, nsabino@cec.iac.br

(3)Universidade Estadual Paulista, Faculdade de Engenharia de Ilha Solteira, Dep. de Fitotecnia, Economia e Sociologia Rural, CEP 15385-000 Ilha Solteira, SP. E-mail: enes@agr.fei.unesp.br

(4)IAC, Centro de Algodão e Fibrosas Diversas. E-mail: mfuzatto@cec.iac.br, lhcarval@iac.br

(5)IAC, Centro de Ação Regional, Caixa Postal 28, CEP 13020-902 Campinas, SP. E-mail: nan.iac@votuporanga.com.br

\section{Introdução}

A cultura do algodão está distribuída em mais de setenta países e em várias regiões do globo terrestre (Abrahão et al., 1982), estimando-se que a produção mundial de fibra seja de aproximadamente $32 \mathrm{mi}-$ lhões de toneladas, sendo que o Brasil produziu em 1996/97 cerca de 307 mil toneladas e importou 460 mil toneladas de fibra (Magalhães, 1998). O consumo brasileiro em 1998 foi em torno de 730 mil toneladas de fibra (Barbosa, 1998), embora haja uma 
projeção de demanda, para o ano 2000, de aproximadamente 1.100.000 toneladas de fibra de algodão para atender o consumo interno e os estoques reguladores (Conselho Nacional da Indústria Têxtil, 1987). Assim, para que a demanda seja atendida com produção própria, o Brasil deveria, no mínimo, duplicar a atual área de plantio, admitindo-se culturas de nível tecnológico adequado.

A expansão da área de cultivo do algodoeiro, como já está sendo verificada em estados da região central do Brasil, deve realizar-se em grandes áreas, possibilitando a colheita mecanizada, principalmente em regiões com baixa disponibilidade de mão-de-obra. A escassez desse fator, a sua má qualidade e seu custo crescente constituem sérios problemas em áreas tradicionais de cultivo, provocando a necessidade de reformulação das técnicas culturais empregadas. A prática do desbaste, recomendada para a cultura do algodoeiro, foi utilizada até 1990. Eram deixadas em torno de cinco plantas por metro de linha de plantio, e isto exigia uma carga de trabalho de, aproximadamente, 17 horas-homem/ha. De acordo com Ferraz et al. (1977), é possível o aumento da densidade de plantas até a eliminação total do desbaste, sem prejuízo para a cultura. Moresco et al. (1999a, 1999b) concluíram, respectivamente, que houve efeito significativo com maior densidade de plantas (12 plantas $/ \mathrm{m}$ ) e com a variedade de algodoeiro utilizada. Por outro lado, Bolonhezi et al. (1997) mostraram que não houve efeito na produção de algodão, nas densidades de 5 e 10 plantas $/ \mathrm{m}$. Carvalho et al. (1999), em estudos de diferentes densidades populacionais ( 7 e 12 plantas/m), não detectaram diferenças significativas para os diversos parâmetros avaliados.

O objetivo deste trabalho foi avaliar o efeito da eliminação de desbaste na cultura do algodoeiro.

\section{Material e Métodos}

Para o estudo, foram conduzidos, durante o período de 1979/80 a 1982/83, onze ensaios em Estações Experimentais do Instituto Agronômico ou em propriedades particulares localizadas em diversas regiões produtoras do Estado de São Paulo. O delineamento experimental foi o de blocos ao acaso, com seis tratamentos e seis repetições. Os tratamentos consistiram de 12 e 24 sementes $/ \mathrm{m}$, submetidas ao deslintamento mecânico; deslintamento mecâ- nico + tratamento com fungicidas; e, deslintamento químico com ácido sulfúrico concentrado + tratamento com fungicidas. $\mathrm{O}$ tratamento com fungicidas consistiu na aplicação de uma mistura de benomyl + thiram $(100 \mathrm{~g}+100 \mathrm{~g}$ de i.a. por $100 \mathrm{~kg}$ de sementes deslintadas mecanicamente e com ácido sulfúrico concentrado). A parcela experimental foi constituída de quatro linhas com $5 \mathrm{~m}$ de comprimento, a espaços de $1 \mathrm{~m}$. Utilizaram-se sementes da variedade comercial de algodão IAC 17, oriundas de campos de aumento do IAC, cuja germinação foi sempre superior a $70 \%$. A adubação por ocasião da semeadura foi de $340 \mathrm{~kg} / \mathrm{ha}$ da fórmula 4-14-8, e em cobertura, com $200 \mathrm{~kg} / \mathrm{ha}$ de sulfato de amônio. As áreas em estudo receberam os tratos culturais recomendados para a cultura do algodoeiro.

A avaliação dos resultados foi feita através das seguintes características: a) porcentagem de emergência - obtida pela relação entre o número de plantas emergidas e o número de sementes semeadas, realizada aos 15-25 dias; b) estande final - número de plantas que produziram algodão, por ocasião da realização da primeira colheita; c) produtividade - colheita do total de algodão produzido na parcela, em $\mathrm{kg} / \mathrm{ha}$; d) porcentagem de fibra - valor médio da sua porcentagem ponderal, obtida após o beneficiamento das amostras; e) pesos de um capulho e de 100 sementes - que correspondem a determinações médias expressas em g; f) índice de fibra - calculado pela fórmula de Christidis \& Harrison (1955): índice de fibra $=(\%$ de fibra $\mathrm{x}$ peso de 100 sementes $) /(100-\%$ de fibra).

Em virtude de as condições de solo e clima terem afetado diferentemente a emergência das plântulas, efetuaram-se dois grupamentos de ensaios: emergência normal quando as condições edafoclimáticas durante e após o plantio foram boas e as plântulas emergiram no período de 5 a 10 dias, considerado normal para a cultura do algodoeiro; e emergência tardia - quando as condições edafoclimáticas após o plantio dos ensaios deste grupamento apresentaram baixos teores de umidade relativa do ar e de temperatura, com precipitação insuficiente ou em excesso, provocando, esta última, maior cobertura de solo no sulco de plantio (compactação superficial), antes da emergência. Tais condições provocaram má germinação das sementes e as plântulas demoraram para emergir (em torno de 14 dias), ficando sujeitas à incidência de patógenos do tombamento.

Em cada local foi feita uma análise estatística por característica estudada, utilizando-se o delineamento estatístico em esquema fatorial: duas densidades ( 12 e 24 sementes $/ \mathrm{m}$ ) e três deslintamentos (mecânico, mecânico + fungicidas e químico + fungicidas). Posteriormente, fo- 
ram feitas as análises conjuntas em esquema fatorial e blocos ao acaso, utilizando-se sete locais para o grupamento de emergência normal e quatro locais para emergência tardia. A comparação das médias foi realizada através do teste de Duncan a $5 \%$ de probabilidade.

\section{Resultados e Discussão}

Na emergência normal, não houve diferença significativa entre 12 e 24 sementes/m, porém na emergência tardia houve um aumento significativo na emergência, quando se utilizou maior número de sementes, independentemente do tratamento utilizado (Tabela 1).

$\mathrm{O}$ uso de fungicidas provocou aumento na emergência nos dois grupamentos, nas duas densidades de plantio, e foi mais pronunciado em emergência tardia.

O deslintamento químico e uso de fungicidas apresentou valores significativamente mais altos do que os obtidos nos demais tratamentos, quando se tratou de emergência normal. Na emergência tardia, este tratamento elevou os valores em relação às sementes só deslintadas mecanicamente, porém mostrou efeito menos pronunciado quando comparado com o tratamento somente com fungicidas. Esse fato foi comprovado estatisticamente (Tabela 1), pela análise conjunta de emergência normal e tardia, onde se obteve um nível significativo para a interação parcial + tardia x tratamento de semente (mecânico, fungicidas, e químico + fungicidas).
Normalmente, sementes deslintadas quimicamente absorvem mais água e germinam mais rapidamente. Em boas condições edafoclimáticas de germinação, deverá ocorrer uma emergência mais rápida, provocando, com isso, melhor estande; porém, em condições adversas, há um período relativamente demorado para a emergência, e as plântulas estarão mais sujeitas ao ataque de fungos do tombamento, provocando, com isso, um efeito negativo no estande inicial de plantas.

Este fato serve de suporte às recomendações técnicas aos cotonicultores que pretendem utilizar plantio sem a operação de raleação, os quais devem fazer a semeadura somente quando o solo apresentar boas condições de germinação. Mesmo que se utilizem sementes de boa qualidade (germinação acima de $70 \%$ ), pode-se chegar a baixo estande na lavoura, em condições edafoclimáticas adversas à germinação.

A viabilidade de eliminar o desbaste na cultura do algodoeiro depende da possibilidade de ser mantida a população adequada de plantas. Isso importa em garantir boa emergência das plântulas e sua sobrevivência até a colheita.

O estande final, em emergência normal, ficou dentro do esperado, de acordo com o número de sementes semeadas, com a qualidade de semente utilizada e a porcentagem de emergência, e detectou-se efeito significativo no tratamento com fungicidas e no deslintamento químico + fungicidas (Tabela 2).

Tabela 1. Emergência normal (5 a 10 dias) e tardia (em torno de 14 dias) de plântulas de algodoeiro, cultivar IAC 17, obtidas a partir de sementes submetidas a diferentes tratamentos ${ }^{(1)}$.

\begin{tabular}{clcc}
\hline $\begin{array}{l}\text { Número de } \\
\text { sementes/m }\end{array}$ & Tratamentos & Emergência normal (\%) & Emergência tardia (\%) \\
\hline 12 & Deslintamento mecânico & $55,12 \mathrm{~d}$ & $24,21 \mathrm{~d}$ \\
12 & Desl. mecânico + fungicida & $60,34 \mathrm{c}$ & $34,21 \mathrm{ab}$ \\
12 & Desl. químico + fungicida & $65,30 \mathrm{ab}$ & $31,23 \mathrm{bc}$ \\
24 & Deslintamento mecânico & $54,41 \mathrm{~d}$ & $27,66 \mathrm{dc}$ \\
24 & Desl. mecânico + fungicida & $62,20 \mathrm{bc}$ & $37,23 \mathrm{a}$ \\
24 & Desl. químico + fungicida & $67,50 \mathrm{a}$ & $34,99 \mathrm{ab}$ \\
\hline $\mathrm{F}$ (tratamentos) ${ }^{(2)}$ & & $17,21^{* *}$ & $8,65^{* *}$ \\
$\mathrm{~F}($ densidade) & & 1,14 & $6,27^{*}$ \\
$\mathrm{~F}($ deslintamento) & & $41,69 * *$ & $18,48^{* *}$ \\
$\mathrm{~F}($ densidade x deslintamento) & 0,77 & 0,02 \\
$\mathrm{CV}(\%)$ & 13,59 & 25,84 \\
\hline
\end{tabular}

${ }^{(1)}$ Médias seguidas pela mesma letra, nas colunas, não diferem estatisticamente pelo teste de Duncan a $5 \%$ de probabilidade. ${ }^{(2)}$ Análise em blocos ao acaso ${ }^{*} \mathrm{e}^{* *}$ Significativo a $5 \%$ e a $1 \%$ de probabilidade, respectivamente 
No caso de emergência tardia, quando se utilizaram 12 sementes $/ \mathrm{m}$, houve uma queda significativa no estande final, mesmo com os tratamentos de fungicidas e deslintamento químico, em relação à utilização de 24 sementes $/ \mathrm{m}$. Esses dados confirmam a tese de que tratamentos químicos e fungicidas nas sementes não são suficientes para um bom estande na lavoura, quando se utiliza pequena quantidade de sementes mediante condições edafoclimáticas desfavoráveis durante a emergência. Nas condições de emergência normal, o plantio de 12 sementes/m proporcionou populações de plantas semelhantes ao observado comumente na lavoura do algodoeiro. Em condições desfavoráveis, o estande foi reduzido praticamente à metade. Esse fato demonstra a possibilidade de se utilizar menor número de sementes no plantio, eliminando-se a operação de desbaste, porém é básico que as condições edafoclimáticas durante a germinação sejam as melhores possível. Os dados mostraram, em média e em condições de emergência normal, que se pode utilizar, no plantio, pequena quantidade de sementes, mas devem ser tratadas com fungicidas e deslintadas quimicamente.

Com respeito à produção de algodão, não houve efeito significativo entre os tratamentos nas duas emergências, embora os estandes tenham variado quanto à emergência normal de 6 a 14 plantas/m, e à emergência tardia de 3 a 7 plantas $/ \mathrm{m}$. Esses resultados não confirmaram os dados apresentados por Moresco et al. (1999a) os quais obtiveram maiores produções em estandes com maior número de plantas (6 a 13 plantas/m). Os estudos da interação variedades $\mathrm{x}$ densidade $\mathrm{x}$ espaçamento realizados por Moresco et al. (1999b) mostraram um aumento de produção em IAC 22 e ITA 90 na densidade de 12 plantas/m e espaçamento de $0,76 \mathrm{~m}$ entre linhas. No espaçamento de $0,90 \mathrm{~m}$, a IAC 22 mostrou menor produção, com 12 plantas $/ \mathrm{m}$.

Em todas as características estudadas, houve efeitos significativos entre 12 e 24 plantas/m, onde menores estandes mostraram melhores resultados (Tabela 3). Talvez este fato seja pela competição entre as plantas ou pelo deslocamento da carga para o terço superior das plantas. Sabe-se que os capulhos dos ponteiros apresentam menores produções e qualidade de fibra inferior e conseqüentemente menores pesos de semente. Resultados semelhantes foram obtidos por Wilkes \& Corley (1968), Hawkins \& Peacock (1970), Bridge et al. (1973) e Ferraz et al. (1977), que mostraram efeitos negativos quanto a essas características com o aumento do estande.

Tabela 2. Estande final e produção de plantas de algodão, cultivar IAC 17, obtidas a partir de plântulas com emergência normal (5 a 10 dias) e tardia (em torno de 14 dias) e de sementes submetidas a diferentes tratamentos ${ }^{(1)}$.

\begin{tabular}{|c|c|c|c|c|c|}
\hline \multirow{2}{*}{$\begin{array}{l}\text { Número de } \\
\text { sementes } / \mathrm{m}\end{array}$} & \multirow[t]{2}{*}{ Tratamentos } & \multicolumn{2}{|c|}{ Emergência normal } & \multicolumn{2}{|c|}{ Emergência tardia } \\
\hline & & $\begin{array}{l}\text { Estande final } \\
\left(\mathrm{n}^{\mathrm{o}} \text { plantas }\right)\end{array}$ & $\begin{array}{c}\text { Produção } \\
\text { (kg/ha) }\end{array}$ & $\begin{array}{l}\text { Estande final } \\
\left(\mathrm{n}^{\mathrm{o}} \text { plantas }\right)\end{array}$ & $\begin{array}{c}\text { Produção } \\
\text { (kg/ha) }\end{array}$ \\
\hline 12 & Deslintamento mecânico & $5,97 \mathrm{~d}$ & 3,19 & $2,71 \mathrm{~d}$ & 2,71 \\
\hline 12 & Deslintamento mec. + fungicida & $6,65 c$ & 3,30 & $4,04 \mathrm{c}$ & 3,04 \\
\hline 12 & Deslintamento químico + fungicida & $7,07 \mathrm{c}$ & 3,34 & $3,50 \mathrm{~cd}$ & 2,84 \\
\hline 24 & Deslintamento mecânico & $11,11 \mathrm{~b}$ & 3,24 & $5,64 \mathrm{~b}$ & 2,96 \\
\hline 24 & Deslintamento mec. + fungicida & $13,25 \mathrm{a}$ & 3,31 & $7,28 \mathrm{a}$ & 2,98 \\
\hline 24 & Deslintamento químico + fungicida & $13,84 \mathrm{a}$ & 3,17 & $7,07 \mathrm{a}$ & 3,07 \\
\hline \multicolumn{2}{|c|}{$\mathrm{F}(\text { tratamentos) })^{(2)}$} & $203,31 * *$ & 0,70 & $38,60 * *$ & 1,53 \\
\hline \multicolumn{2}{|c|}{ F (densidade) } & $1120,50 * *$ & 0,29 & $166,77 * *$ & 2,43 \\
\hline \multicolumn{2}{|c|}{ F (deslintamento) } & $38,7 * *$ & 0,61 & $12,56 * *$ & 1,42 \\
\hline \multicolumn{2}{|c|}{$\mathrm{F}$ (densidade $\mathrm{x}$ deslintamento) } & $7,76 * *$ & 0,95 & 0,56 & 1,20 \\
\hline \multicolumn{2}{|c|}{$\mathrm{CV}(\%)$} & 15,16 & 16,00 & 29,94 & 17,61 \\
\hline
\end{tabular}




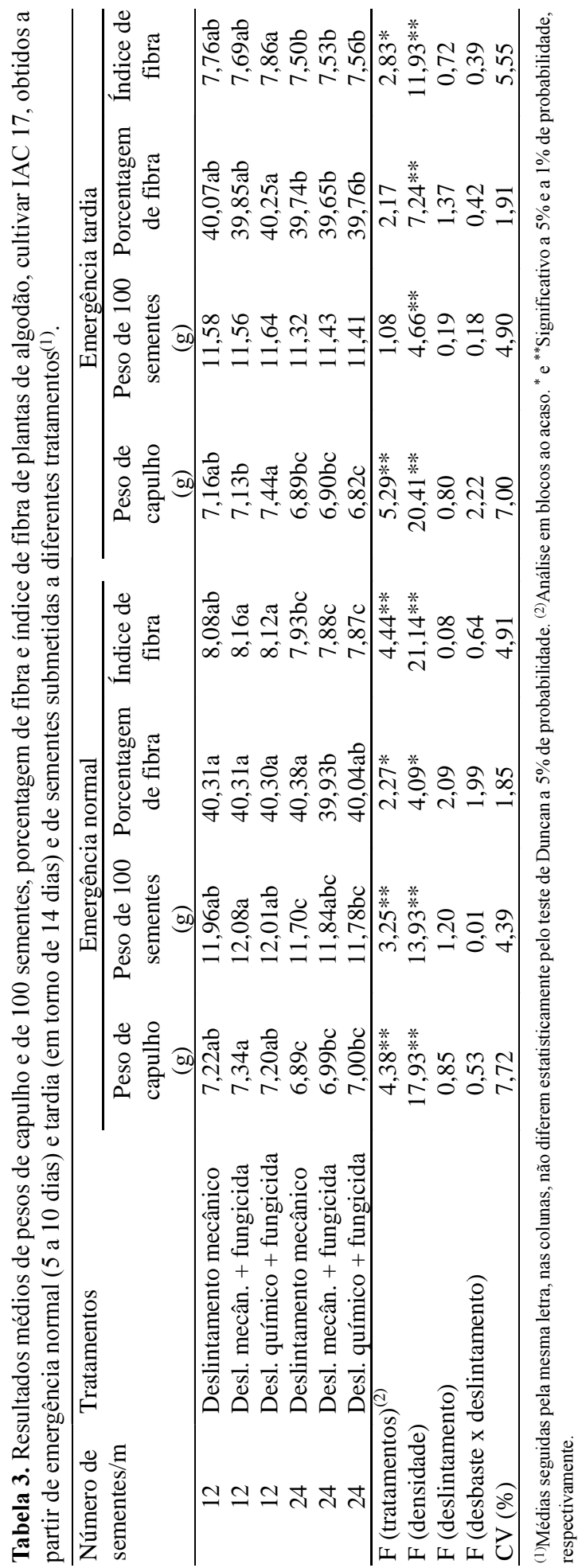

À medida que se diminuiu o peso de capulho e de sementes, era de se esperar uma maior porcentagem de fibra, fato que, em média, não ocorreu. Onde houve maior estande, ocorreu pequena redução nos pesos de 100 sementes e de capulho e da porcentagem de fibra, fato esse já assinalado por Wilkes \& Corley (1968). Tal redução poderia ser explicada pela diminuição do índice de fibra, também notada por Christidis \& Harrison (1955). A redução desse índice ocorreu pela diminuição do índice de maturidade da fibra, devido à insolação inadequada às plantas ou pela elevação da carga na parte superior da planta, provocando, com isso, menor deposição de celulose na parede secundária da fibra.

\section{Conclusões}

1. É possível suprimir o desbaste na lavoura de algodão, através da utilização do tratamento das sementes com fungicida, ou deslintá-las quimicamente.

2. A utilização de menor número de sementes por área de plantio requer, sempre, boas condições edafoclimáticas para germinação, aliadas ao tratamento com fungicida e deslintamento químico.

3. Em condições desfavoráveis à emergência, consegue-se melhor estande, utilizando-se maior número de sementes tratadas com fungicidas.

4. Com maior estande de plantas ocorre diminuição do peso do capulho e das sementes, da porcentagem de fibra e do índice de fibra.

\section{Referências}

ABRAHÃO, J. T. M.; D'ARCE, M. A. B. R.; FONSECA, H. Algodão: produção, pré-processamento e transformação industrial. São Paulo: Secretaria da Indústria, Comércio, Ciência e Tecnologia, 1982. 96 p. (Série Extensão Agroindustrial, 2).

BARBOSA, M. Z. Algodão. Prognóstico Agrícola, São Paulo, v. 2, p. 151-156, 1998.

BOLONHEZI, A. C.; TORAVETI, C. R.; BOLONHEZI, D. Comportamento do algodoeiro herbáceo 'IAC 20' submetido a diversos espaçamentos. In: CONGRESSO BRASILEIRO DE ALGODÃO, 1., 1997, Fortaleza. Anais... Campina Grande: Embrapa-CNPA, 1997. p. 552-554. 
BRIDGE, R. R.; MEREDITH JUNIOR, W. R.; CHISM, J. F. Influence of planting method and plant population on cotton (Gossypium hirsutum L.). Agronomy Journal, Madison, v. 65, p. 104-109, 1973.

CARVAlHO, L. H.; CHIAVEGATO, E. J.; CIA, E.; SILVA, N. M.; SABINO, N. P.; KONDO, J. I.; DINARDOMIRANDA, L. L. Comportamento de cultivares em diferentes densidades populacionais, na presença de nematóides. In: CONGRESSO BRASILEIRO DE ALGODÃO, 2., 1999, Ribeirão Preto. Anais... Campina Grande: Embrapa-CNPA, 1999. p. 626-628.

CHRISTIDIS, B. G.; HARRISON, G. J. Cotton growing problems. New York: McGraw-Hill, 1955. 633 p.

CONSELHO NACIONAL DA INDÚSTRIATÊXTIL (Rio de Janeiro, RJ). Diretrizes e recomendações para formulação de uma política de incentivo à produção e à melhoria da qualidade do algodão brasileiro. Rio de Janeiro: Senai/Centro de Tecnologia da Indústria Química e Têxtil, 1987. 45 p.

FERRAZ, C. A. M.; CIA, E.; SABINO, N. P.; GROSSI J. M. M.; VEIGA, A. A.; YOSHIDA, H. Efeitos da densidade de plantio e da aplicação de CCC em algodoeiro. Bragantia, Campinas, v. 36, p. 239-251, 1977.
HAWKINS, B. S.; PEACOCK, H. A. Yield response of upland cotton (Gossypium hirsutum L.) to several spacing arrangements. Agronomy Journal, Madison, v. 62, p. 578580, 1970.

MAGALHÃES, J. S. B. Mercado de algodão: perspectivas no Brasil e no mundo. In: ENCONTRO SOBRE A CULTURA DO ALGODOEIRO, 1997, Ituverava. Anais... Jaboticabal: Funep, 1998. p. 121-134.

MORESCO, E. R.; FARIAS, F. J. C.; SOUZA, M.; AGUIAR, P. H.; MARQUES, M. F.; TAKEDA,C. Influência da densidade e do espaçamento na produtividade do algodoeiro herbáceo. In: CONGRESSO BRASILEIRO DE ALGODÃO, 2., 1999, Ribeirão Preto. Anais... Campina Grande: Embrapa-CNPA, 1999a. p. 629-631.

MORESCO, E. R.; FARIAS, F. J. C.; SOUZA, M.; MARQUES, M. F.; TAKEDA, C. Influência da densidade e do espaçamento na produtividade do algodoeiro herbáceo I. In: CONGRESSO BRASILEIRO DE ALGODÃO, 2., 1999, Ribeirão Preto. Anais... Campina Grande: EmbrapaCNPA, 1999b. p. 632-633.

WILKES, L. H.; CORLEY, T. E. Planting and cultivation. In: ELLIOT, F. E.; HOOVER, M.; PORTER JUNIOR, W. K. (Ed.). Advances in production and utilization of quality 'cotton': principles and practices. Ames: Iowa State University Press, 1968. p. 115-117. 